\title{
Vhl deficiency in osteocytes produces high bone mass and hematopoietic defects
}

Gabriela G. Loots ${ }^{1,2}$, Alexander G. Robling ${ }^{3}$, Jiun C. Chang ${ }^{1,2}$, Deepa K. Murugesh ${ }^{1}$, Jamila Bajwa ${ }^{2}$, Cameron Carlisle $^{2}$, Jennifer O. Manilay ${ }^{2}$, Alice Wong ${ }^{4}$, Clare E. Yellowley ${ }^{4}$, and Damian C. Genetos ${ }^{4}$

${ }^{1}$ Lawrence Livermore National Laboratories, Physical and Life Sciences Directorate, Livermore, CA.

${ }^{2}$ Molecular Cell Biology Unit, School of Natural Sciences, UC Merced, Merced, CA.

${ }^{3}$ Department of Anatomy and Cell Biology, Indiana University School of Medicine, Indianapolis, IN, USA.

${ }^{4}$ Department of Anatomy, Physiology, and Cell Biology, School of Veterinary Medicine, University of California Davis, Davis, CA, USA.

\section{Corresponding author:}

Damian C. Genetos

Department of Anatomy, Physiology and Cell Biology

School of Veterinary Medicine

1089 Veterinary Medicine Drive

University of California Davis, Davis CA 95616

Email: dgenetos@ucdavis.edu

This is the author's manuscript of the article published in final edited form as:

Loots, G. G., Robling, A. G., Chang, J. C., Murugesh, D. K., Bajwa, J., Carlisle, C., ... Genetos, D. C. (2018). Vhl deficiency in osteocytes produces high bone mass and hematopoietic defects. Bone, 116, 307-314. 


\section{ABSTRACT}

Tissue oxygen $\left(\mathrm{O}_{2}\right)$ levels vary during development and disease; adaptations to decreased $\mathrm{O}_{2}$ (hypoxia) are mediated by hypoxia-inducible factor (HIF) transcription factors. HIFs are active in the skeleton, and stabilizing HIF- $\alpha$ isoforms cause high bone mass (HBM) phenotypes. A fundamental limitation of previous studies examining the obligate role for HIF- $\alpha$ isoforms in the skeleton involves the persistence of gene deletion as osteolineage cells differentiate into osteocytes. Because osteocytes orchestrate skeletal development and homeostasis, we evaluated the influence of $\mathrm{Vhl}$ or Hif1a disruption in osteocytes. Osteocytic Vhl deletion caused HBM phenotype, but Hif1a was dispensable in osteocytes. Vh/ cKO mice revealed enhanced canonical Wnt signaling. B cell development was reduced while myelopoiesis increased in osteocytic $\mathrm{Vhl}$ cKO, revealing a novel influence of $\mathrm{Vhl} / \mathrm{HIF}-\alpha$ function in osteocytes on maintenance of bone microarchitecture via canonical Wnt signaling and effects on hematopoiesis.

\section{KEYWORDS:}

Osteocyte

von Hippel-Landau

Hypoxia-inducible factor

Wnt

Sclerostin

hematopoiesis 


\section{INTRODUCTION}

Oxygen $\left(\mathrm{O}_{2}\right)$ levels vary throughout the body. They change spatiotemporally during development, and can be acutely impacted during pathophysiological insults, such as stroke or bone fracture. Adaptive responses to decreased $\mathrm{O}_{2}$ (hypoxia) are rectified by transcriptional induction of genes promoting angiogenesis and anaerobic glycolysis, which are mediated, in part, by the hypoxia-inducible factor (HIF) family of transcription factors[1]. Constitutively expressed HIF- $\beta$ subunits bind to one of three distinct HIF- $\alpha$ isoforms (HIF-1 $\alpha$, HIF-2 $\alpha$, and HIF-3 $\alpha$ ) to initiate gene expression, although HIF-3 $\alpha$ is considered an inhibitor of HIF-1 $\alpha$ and HIF-2 $\alpha$. Oxygen levels regulate HIF-responsive gene expression. Under normoxic conditions, HIF- $\alpha$ subunits are proline hydroxylated by proline hydroxylase-domain proteins (PHDs), targeted for ubiquitination by the E3 ligase complex von Hippel-Lindau (Vhl) and are ultimately degraded by the $26 S$ proteasome. Under hypoxic conditions, prolyl hydroxylation of HIF- $\alpha$ is inhibited, enabling HIF- $\alpha$ to accumulate, translocate into the nucleus and complex with HIF- $\beta / A R N T$, and ultimately bind to HIF-response elements (HRE) within target genes.

The VHL/HIF system is highly active in the skeleton. Multiple strategies that use osteolineagespecific cre drivers and deletion of PHD or Vhl to stabilize HIF-1 $\alpha$ and HIF-2 $\alpha$ increase bone mass. Individually or combinatorially deleting Phd1, Phd2, or Phd3 in Osterix-positive osteoprogenitors improves trabecular microarchitecture dependent upon the degree of HIF- $\alpha$ stabilization[2]. Similarly, deletion of $\mathrm{Vhl}$ in mature osteoblasts using an osteocalcin (Bglap) cre driver increases trabecular and cortical microarchitecture[3].

The murine models described above clearly demonstrate that manipulating HIF- $\alpha$ levels in bone cells influences skeletal development and homeostasis. In these models, $\mathrm{Vhl}$ or Phd isoform elimination persists as osteoprogenitors differentiate into osteoblasts and then transition into osteocytes. Thus, these models cannot elucidate the influence of $\mathrm{Vhl}$ deletion in osteocytes compared to $\mathrm{Vhl}$ deletion in osteoprogenitors or osteoblasts. Osteocytes are the most abundant cells within bone, comprising $>90 \%$ of total cells[4]. Osteocytes exert critical roles in mechano-sensing, skeletal remodeling, mineral homeostasis and hematopoiesis[5]. Indeed, the osteocyte and the extensive osteocytic network in bone is now recognized as a central regulator of skeletal activity $[5,6]$. The frequency of HIF-1 $1 \alpha$-positive osteocytes increases in mice under disuse conditions[7]; in vitro, exposing osteocyte-like cells to hypoxia augments secretion of chemotactic factors[8] and GDF15 to promote osteoclastogenesis[9], and influences the osteoblast-to-osteocyte transition[10]. Other osteocytic functions attributed to HIF- $\alpha$ function include directing appropriate bone formation in response to mechanical loading[11], sensing microdamage[12], and development of the lacunocanalicular network in which osteocytes reside[13].

Provided the recognition that osteocytes orchestrate many aspects of skeletal development and homeostasis, we sought to evaluate the influence of $\mathrm{Vhl}$ or Hif1a disruption in osteocytes. We used a cre-loxP system under control of the non-inducible 10kb-Dmp1 promoter[14] to address the function of $V h l$ in osteocytes. Similar to results using Bglap-cre to delete $V h /$ in mature osteoblasts[3], osteocytic deletion of $\mathrm{Vh} /$ produced mice with a high bone mass (HBM) phenotype that dramatically increased trabecular and cortical microarchitecture. Femoral osteocytes in $\mathrm{Vhl}$ cKO mice revealed qualitative and quantitative reductions in sclerostin immunostaining, and concomitant increases in activated $\beta$ catenin levels, suggesting that Vhl/HIF- $\alpha$ signaling influences canonical Wnt signaling in osteocytes. Vhl cKO mice mated to mice lacking the Wnt co-receptor Lrp5 or to transgenic mice over-expressing the human SOST gene revealed that $V h l$ functions epistatically to canonical Wnt signaling: cortical microarchitecture resolved to wild-type phenotype in compound mice, but not trabecular microarchitecture. Osteocytic HIF- $\alpha$ contributes to hematopoiesis, as B cell development is blocked in osteocytic $\mathrm{Vhl}$ cKO mice. These data reveal a novel influence of Vhl/HIF- $\alpha$ function in osteocytes on maintenance of bone microarchitecture via canonical Wnt signaling and effects on hematopoiesis. 


\section{MATERIALS AND METHODS}

Mice

Vhlh $h^{f / f}[15](\# 004081)$ and Hif1 ${ }^{\mathrm{f} / \mathrm{f}}[16](\# 007561)$ were purchased from Jackson Laboratories and bred to control C57BI/6 or with mice expressing cre recombinase from the 10kb-Dmp1 promoter (Dmp1-cre) [B6N.FVB-Tg(Dmp1-cre)1Jqfe/BwdJ] [14] (kindly provided by Dr. Jian Q. Feng, Department of Biomedical Sciences, Texas A\&M University Baylor College of Dentistry) to generate Dmp1-cre; Vhlh ${ }^{f / f}$ and Dmp1-cre; $H_{i f 1 a^{f / f}}$ mice with osteocyte conditional deletion of Vhlh (hereafter, Vhl cKO) or Hif1a (Hif1a cKO). Mice globally deficient for Lrp5 have been previously described ( $L r p 5^{t m 1.2 V a r i} ;$ kindly provided by Dr. B. Williams[17]); mice over-expressing human SOST under control of a BAC have been described previously [18,19]. All animal experimental procedures were completed under an approved protocol by the IACUC committees at Lawrence Livermore National Laboratory and University of California, Merced and conform to the NIH Guide for the Care and Use of Laboratory Animals. All animals used in this study were 3.5-4 months of age at analysis.

\section{Immunocytochemistry, histology, and immunohistochemistry}

Femur and L4 lumbar vertebrae were collected at 16 weeks of age from male mice, and fixed, dehydrated, embedded and sectioned as previously described[18]. Detailed description of immunocytochemistry is available in Supplemental Information. Hematoxylin and eosin (Sigma) staining was performed as in [19]; MacNeal's tetrachrome/Von Kossa staining and toluidine blue staining were performed as described in [20]. Dynamic histomorphometry was performed and analyzed as in[21].

\section{Micro-computed tomography $(\mu C T)$}

At euthanasia, the right femur was extracted, placed in $10 \%$ neutral-buffered formalin for two days, and then stored in $70 \%$ ethanol at $4^{\circ} \mathrm{C}$. A 2.6- $\mathrm{mm}$ span of the distal femoral metaphysis was scanned on a high resolution $\mu \mathrm{CT}$ ( $\mu \mathrm{CT}$ 35; Scanco Medical AG) at $13 \mu \mathrm{m}$ resolution using 50kV peak tube potential and $151 \mathrm{~ms}$ integration time to measure trabecular three-dimensional morphometric properties as described in [21].

Isolation of Bone Marrow Cells and Splenocytes and Flow Cytometry

Bone marrow cells and splenocytes were isolated as in[22]. Both BM cell and splenocyte suspensions were filtered through 70 micron nylon mesh squares into clean $15 \mathrm{ml}$ conical tubes. Total cell counts were obtained using a hemocytometer and Trypan Blue staining to exclude dead cells. Detailed description of antibodies and staining parameters are available in Supplemental Information. Cells were acquired on a Becton Dickinson LSR II and flow cytometric analysis was performed using FlowJo software.

\section{Statistical Analysis}

All data were expressed as the mean \pm standard deviation. Unpaired Student's t-test, or one-way ANOVA followed by Fisher's LSD test were used where appropriate. For flow cytometry results, statistical analysis was done using Student's t-test with a two-tailed distribution, with two-sample equal variance (homoscedastic test). For all tests, $p<0.05$ was considered statistically significant. 


\section{RESULTS}

Deletion of $\mathrm{Vhl}$ in osteocytes has robust effects on trabecular and cortical microarchitecture

Deletion of $\mathrm{Vhl}$ and subsequent stabilization of HIF-1 $\alpha$ and HIF-2 $\alpha$ in mature osteoblasts promotes bone modeling and accrual, whereas deletion of Hif1a produces an inverse skeletal phenotype[3]. Because Vhl deletion in mature osteoblasts persists as osteoblasts differentiate into osteocytes, previous data do not identify the impact of osteocytic $\mathrm{Vh} /$ deletion on skeletal mass and architecture. In order to examine the impact of $\mathrm{Vhl}$ and Hif1a expression in osteocytes, we used a cre-loxP system wherein cre recombinase was expressed under control of the $10 \mathrm{~kb}-D m p 1$ promoter[14]. Immunofluorescent staining of Vhl was decreased in sections from $\mathrm{Vhl}$ cKO mice compared to cre-negative control mice (Figure 1A); conversely, staining for HIF-1 $\alpha$ or HIF-2 $\alpha$ was more robust in $V h l$ cKO sections. The fraction of osteocytes staining positively for HIF-1 $\alpha$ or HIF-2 ain $\mathrm{Vhl}$ cKO animals (Figure 1C) increased; consistent with observations by Wu et al.[2], not all osteocytes were immunoreactive for HIF-1 $\alpha$ or HIF-2 $\alpha$.

Hematoxylin and eosin staining revealed gross changes in cortical and trabecular phenotype in $\mathrm{Vhl}$ cKO mice compared to cre-negative control mice in both the appendicular (Figure 2A) and axial (Supplemental Figure 1B) skeleton. $\mathrm{Vhl}$ cKO bones showed extensive endomucin immunoreactivity (Figure 2B), indicating increased vascularization. Sagittal and transverse microCT sections of the distal femur revealed a robust increase in trabecular bone volume fraction in $\mathrm{Vhl}$ cKO mice compared to wild-type mice (Figures 2C and 2D); trabecular thickness (Figure 2E) and number (S2A) were also increased in $\mathrm{Vhl}$ cKO mice, with concomitant decreases in trabecular spacing (S2B). Cortical microarchitecture was also influenced by $\mathrm{Vhl}$ deletion in osteocytes. Total cross-sectional area (Tt.Ar, Figure 2F), cortical bone area (Ct.Ar; Figure 2G), and cortical thickness (Figure $\mathbf{2 H}$ ) each increased in cKO mice relative to cre-negative mice. Polar moment of inertia, which quantifies the expected resistance to torsional loads, also increased in Vhl cKO mice (Figure 2I).

The severe HBM phenotype observed in osteocytic $\mathrm{Vhl}$ cKO mice prompted us to evaluate the mechanism of rampant bone accumulation in order to determine whether the phenotype is driven largely by a deficiency in osteoclastic bone resorption. Von Kossa/MacNeal's tetrachrome staining of the distal femur revealed calcified matrix (bone or cartilage) in osteocytic $\mathrm{Vhl}$ cKO compared to cre-negative mice (S2D). Toluidine blue staining showed no evidence of residual calcified cartilage (Figure S2E), a hallmark of osteoclastic defects as sin many forms of osteopetrosis. In contrast, the pale blue staining of the mineralized portion of the metaphysis indicates that the cancellous bone comprises secondary, remodeled bone. In contrast, note the dark blue staining of the epiphyseal plate (S2E). These data demonstrate that manipulating HIF- $\alpha$ function in osteocytes exerts a strong influence on skeletal microarchitecture and vascularity.

Osteocytic Hif1a does not contribute to skeletal development.

Previous works examining the influence of HIF- $\alpha$ function in bone have reported an inverse phenotype in animals lacking $\mathrm{Vh} /$ compared to animals lacking HIF- $\alpha$ isoforms. Where Bglap-cre; Vh/h/f animals have a high bone mass phenotype, Bglap-cre;Hif1a ${ }^{f / t}$ animals have low bone mass and inferior skeletal microarchitecture [3]. Because $\mathrm{Vhl}$ cKO mice displayed a high bone mass phenotype, we expected mice lacking Hif1a in osteocytes to have an osteoporotic phenotype. Unexpectedly, we found that Dmp1-cre; Hif1a ${ }^{t / f}$ mice were phenotypically indistinguishable from cre-negative control mice (Figure 3A), with no significant differences in trabecular bone volume fraction (Figure 3B), cortical area fraction (Figure 3C), or bone formation rate (Figure 3D). The absence of a skeletal phenotype in osteocytic Hif1a cKO mice, compared to the reported low bone mass phenotype of osteoblastic Hif1a cKO mice[3], suggests that osteocytes utilize an alternate HIF- $\alpha$ isoform, or HIF-independent signaling, to influence the skeleton.

\section{Osteocytic Vhl regulates canonical Wnt signaling}

Activation of Wnt signaling occurs during load-induced bone formation[23] and is required for new bone deposition in response to external loads[24,25]. Although osteocytes do not directly contribute to bone formation, osteocytic $\beta$-catenin is required for the osteoanabolic effect of mechanical loading[26], and constitutively active $\beta$-catenin in osteocytes increases both cortical and trabecular bone mass through increasing both arms of remodeling[27]. To identify a role for Wnt signaling in the observed HBM phenotype, we evaluated Sclerostin and activated $\beta$-catenin levels in decalcified sections of cre-negative and $\mathrm{Vhl} \mathrm{cKO}$ femora by immunocytochemistry. Sclerostin immunoreactivity was markedly decreased in $\mathrm{Vhl}$ cKO osteocytes compared to cre-negative mice (Figures 4A and S5A), whereas there was a concomitant increase in osteocytes staining for activated $\beta$-catenin, suggesting that osteocytic $\mathrm{Vhl}$ could function epistatically to reciprocally regulate Sclerostin expression and canonical Wnt signaling. 
To determine if Vhl-mediated changes in Wnt signaling were correlative or causative for the observed HBM phenotype, we mated $\mathrm{Vhl}$ cKO mice to mice with impaired Wnt signaling, achieved via SOST over-expression $\left(S O S T^{T G}\right)$ [28] or Lrp5 deletion $\left(\mathrm{Lrp5}^{-}\right)$[25]. Both Lrp5 deficiency and SOST overexpression blunted the HBM phenotype of $\mathrm{Vhl}$ cKO but failed to fully resolve the HBM phenotype (Figure 4B). Compound Vhl/Wnt mutant mice revealed compartment-specific restoration of skeletal microarchitecture, wherein trabecular microarchitecture in compound mice had an intermediate HBM phenotype (Figures 4B-4D; S5B and S5C); in contrast, the cortical microarchitecture was indistinguishable from wild-type mice, (Figures 4B, 4E, and 4F; S5D and S5E). These results suggest that trabecular anabolic effects of $V h /$ deficiency do not exclusively depend on Wnt signaling via the Lrp5 co-receptor, but may require additional Wnt-signaling receptors/coreceptors, or may occur via non-canonical mechanisms.

\section{Cell-extrinsic effects of osteocytic Vhl deletion on hematopoiesis}

The integrity of the bone marrow microenvironment is critical for the maintenance of hematopoietic stem cells and their differentation into specific immune cell types and erthryocytes. The severely reduced bone marrow cavity volume of the $\mathrm{Vhl}$ cKO (Figure 5A) is similar to the phenotype observed in Sost ${ }^{-1-}$ [22] and Prx1cre; Sost $t^{f / 4}$ 29] mice. Absolute numbers of total bone marrow cells (Supplemental Table 1), CD45 ${ }^{+}$ hematopoietic cells (Figure 5B), and total CD19 $\mathrm{B}$ lymphocytes (Supplemental Table 1) were significantly reduced in the marrow of the $\mathrm{Vhl}$ cKO mice. B cell maturation-distinguished by cell surface expression of

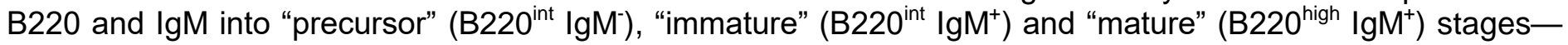
was decreased in bone marrow of $\mathrm{Vhl}$ cKO mice (Figures 5C and 5D). Further, we observed greater proportions of monocytes and granulocytes and an overall reduction in the numbers of all hematopoietic lineages in the bone marrow of $\mathrm{Vhl}$ cKO mice (TABLE 1). Total splenocyte number and distributions of hematopoietic lineage cells were altered in the spleen of $V h l$ cKO mice (Supplemental Table 2). The proportion of LSK (Lineage-negative Sca1 ${ }^{\text {high }} \mathrm{cKit}^{\text {high }}$ ) hematopoietic progenitors increased in both the bone marrow and the spleens of $\mathrm{Vhl}$ cKO mice (Figure 5E), whereas the absolute numbers of LSKs in the bone marrow of cre-negative and $\mathrm{Vhl}$ cKO mice were similar (S6B and S6C).

Although Vhl deletion was targeted to the bone, B cell development in the spleen were affected by osteocytic $\mathrm{Vh} /$ deletion. Total splenocyte number and distributions of hematopoietic lineage cells were altered in the spleen of $\mathrm{Vhl} / \mathrm{cKO}$ mice (Supplemental Table 2). Splenic CD19 ${ }^{\text {high }} \mathrm{B} 220^{\text {high }}$ cells were reduced relative to control animals (Figure 5F), indicating a reduction of mature B cells. However, further investigation with additional cell surface markers demonstrated that within mature splenic B cells, there was an increase in the proportion of $\operatorname{lgM}^{+} \lg D^{\text {low }}$ cells (Figure 5G), indicating an immature phenotype. Evaluating CD19 ${ }^{\text {int }} B 220^{\text {int }}$ populations revealed cells in $\mathrm{Vhl}$ cKO spleens expressing an $\lg M+\lg D^{\text {low }}$ staining pattern (Figure 5G), reminiscent of the staining pattern found on plasma B cells and the "natural antibody" producing innate-like B-1 B cells, which are normally not found in the spleen [30]. Taken together, our data demonstrate that osteocytic $\mathrm{Vhl}$ deletion has dramatic effects on hematopoiesis in the bone marrow, and suggests that these changes in the bone marrow result in major effects on hematopoiesis and B cell development in the spleen. 


\section{DISCUSSION}

\section{Osteocytic Vhl contributes to skeletal development.}

Stabilizing HIF- $\alpha$ isoforms, via deletion of Phd1, Phd2, or Phd3[2], or Vh/[3], produced HBM mice, which affected both trabecular and cortical bone. Osteoblastic $\mathrm{Vh} /$ deletion also increased bone formation in response to distraction osteogenesis[31], and pharmacologic activation of HIF- $\alpha$ s increased bone volume in distraction gaps[31,32] and fracture callus[33]. Thus, using genetic and pharmacologic means, it is clear that stabilizing HIF- $\alpha$ isoforms influences skeletal microarchitecture, demonstrating the fundamental requirement for HIF- $\alpha$ isoforms in skeletal development, homeostasis, and repair. Yet, how osteocytes, embedded within the skeleton, use pericellular oxygen levels and HIF- $\alpha$ stabilization as a guide for skeletal homeostasis remains unexamined. Further, deleting proteins in osteoprogenitors or osteoblasts that regulate HIF- $\alpha$ stability, such as Vhl[3] or PHD isoforms[2], creates osteocytes deficient in said proteins; thus, previous studies have not been able to evaluate the contribution of osteocytic HIF- $\alpha$ signaling toward skeletal development. While there are reports of $10 \mathrm{~kb}$-Dmp1-cre expression in mature osteoblasts[34] and non-skeletal tissues (Pdfgra-expressing gastrointestinal mesenchyme, and portions of the hindbrain)[35], and this study did not evaluate the potential role of non-skeletal Dmp1-cre expression and Vhl excision on the observed skeletal phenotype. With respect to Dmp1-cre expression in osteoblasts, we find no skeletal phenotype in Dmp1-cre; Hif1a cKO mice, in contrast to work using Bglap-cre; Hifa cKO mice, suggesting that modest Dmp1-cre activity in osteoblasts is inadequate to counterbalance the effect of excision in osteocytes. Thus, our results demonstrate that HIF- $\alpha$ signaling in osteocytes has tremendous impact on skeletal development, recapitulating the high bone mass phenotype observed when $\mathrm{Vh} /$ is deleted in mature osteoblasts[3].

\section{Differential requirement for HIF- $\alpha$ isoforms in osteolineage cells}

Osteocytic Vhl deletion stabilizes HIF- $\alpha$ isoforms and produces a HBM phenotype. Thus, we anticipated that osteocytic Hif1a deletion would produce an inverse phenotype, as occurs in mice in which Vhl vs. Hif1a are deleted in mature osteoblasts under control of the Bglap-cre driver[3]. In stark contrast to these results, osteocytic Hif1a cKO mice showed no skeletal phenotype, and were phenotypically identical to cre-negative mice. Although not conclusive, these results suggest that osteocytes utilize an alternate HIF- $\alpha$ isoformspecifically HIF-2 $\alpha$-to produce the HBM phenotype observed in osteocytic $\mathrm{Vhl}$ cKO mice. Indeed, our results and other results in osteoprogenitors and mature osteoblasts suggest that HIF- $\alpha$ isoforms are differentially required by osteoprogenitors, mature osteoblasts, and osteocytes. Deleting Phds or $\mathrm{Vhl}$ increases cortical and trabecular bone in each model [2,32], and our results, but deletion of Hif1a or Hif2a exerts osteolineagespecific effects on the skeleton. In osteoprogenitors (Osx-cre), individual deletion of Hif1a or Hif2a had no effect on trabecular bone[2]. In mature osteoblasts (Bglap-cre), deleting Hif1a decreased trabecular bone volume fraction (Tb.BV/TV) and cortical cross-sectional area; Hif2a deletion moderately affected trabecular or cortical bone[36]. We observe a high bone mass (HBM) phenotype in osteocytic Vhl cKO, but absence of an inverse phenotype in osteocytic Hif1a cKO mice. This scenario is similar to what is observed during the osteoblast-to-osteocyte transition[34,37]; indeed, Hif1a transcript decreases 55\% during the osteoblast-toosteocyte transition, whereas Hif2a levels increase 265\% (data from GSE54784[37]). Alternately, HIF- $\alpha$ independent functions of $\mathrm{Vhl}$, such as stabilization of $\mathrm{Ndrg} 3[38]$, may contribute to the $\mathrm{Vhl} \mathrm{cKO} \mathrm{HBM}$ phenotype..

\section{Functional integration of $\mathrm{Vhl}$ and Wht signaling}

Both Vhl/HIF- $\alpha[3]$ and Wnt/Lrp5[39] signaling robustly influence skeletal development. Our results suggest that these osteoanabolic pathways are epistatic: $\mathrm{Vhl}$ cKO animals demonstrate decreased immunostaining for the Wnt receptor antagonist Sclerostin and concomitant increases in levels of activated $\beta$-catenin. We have previously reported similar results in vitro, wherein culturing clonal mature osteoblasts similarly decreases Sost transcript levels and increases activated $\beta$-catenin levels[40]. Yet, the epistatic relationship that we report within is likely highly context-dependent, depending on, for example, the cre driver used and the osteogenic differentiation state of the cells in which oxygen sensing is manipulated. For example, Riddle et al. reported that HIF-1 $\alpha$ antagonizes load-induced $\beta$-catenin signaling[11], and hypoxic induction of MC3T3-E1 preosteoblasts induces Sclerostin expression to reduce Wnt signaling[41]. Similarly, $V h l$ deletion in osteochondroprogenitors (Col2a1-cre ${ }^{E R T 2}$ ) has an osteoanabolic effect on trabecular, but not cortical, bone[42].

We observe skeletal compartment-specific influence of $\mathrm{Vhl}$ deletion on Wht signaling. In both models $\mathrm{Vhl}$ $\mathrm{cKO}$; $\mathrm{Lrp5}^{\prime-}$ or $\mathrm{Vhl} \mathrm{cKO}$; SOST ${ }^{T G}$ mice, cortical microarchitecture is phenotypically normalized to mirror the 
microarchitecture of wild-type mice, whereas trabecular phenotype is partially rescued. Compartment-specific effects of Wnt signaling have previously been reported: Wnt $16^{-/}$mice have decreased cortical, but not trabecular bone mass[43]; additionally, over-expression of the Wnt antagonist sFrp4 decreases trabecular microarchitecture without affecting cortical mass or geometry[44]. The fundamental cause of compartmentspecific effects on microarchitecture remain unknown, and are attributed to differential expression of Wnt ligands or antagonists[43], turnover rate, and proximity to the bone marrow niche and vascular network. Normalization of cortical microarchitecture in Vhl/Wnt compound mutant mice suggests that Vhl signaling is more fully epistatic in cortical bone than in trabecular bone.

\section{Osteocytic regulation of hematopoiesis}

Osteoblasts and osteocytes regulate hematopoiesis. Mice expressing a constitutively-active parathyroid hormone $(\mathrm{PTH}) / \mathrm{PTH}$-related peptide $(\mathrm{PTHrP})$ receptor PTH1R under control of the type I collagen 2.3kb promoter have increased LSK population of hematopoietic stem cells in bone marrow[45]. In the absence of genetic manipulation, administering PTH to calvarial explants in vitro increases B lymphopoiesis [46]. Conversely, disrupting $\mathrm{G}_{\mathrm{s}}$-alpha subunit signaling in pre-osteoblasts[47], or conditional osteoblast ablation[46], decreases B cell progenitors. In osteocytes, Gs $\alpha$ signaling regulates myelopoiesis, demonstrated through increased granulocytes and platelets in the peripheral blood, as well as splenomegaly[48]; this occurs independently of osteocytic PTH1R signaling, as such transgenic mice reveal no HSC abnormalities[49]. Similarly, osteocytic RANKL (Tnfsf11) is necessary for ovariectomy-induced B cell expansion[50], and osteocyte ablation decreases B lymphopoiesis and thymocyte depletion[51]. Our data suggest that the $\mathrm{Vh} / \mathrm{cKO}$ microenvironment skews HSCs to the myeloid lineages at the expense of lymphoid development. T lymphocyte development appears normal, and only B cell development is affected in Vhl cKO mice. Eludication of the molecular programs within osteocytes that drive hematopoiesis require extensive analysis of purified adult hematopoietic niche cells such as mesenchymal stem cells, osteoblasts and endothelial cells[52]. Taken together, these data suggest that structural aspects of the bone are important in the regulation of hematopoietic stem cells and immune cell development, but the specific role of $\mathrm{Vh} /$ deletion osteocyte function, and how this alters the function of HSCs, B cell progenitors and progeny is still unknown. Further studies are required to determine the molecular mechanisms underlying the effects of acute bone remodeling on hematopoiesis. 


\section{ACKNOWLEDGEMENTS}

Research reported in this publication was supported by the University of California Merced faculty research awards (JOM), Veterans Administration award number BX001478 (to A.G.R) and the National Institute of Arthritis and Musculoskeletal and Skin Diseases of the National Institutes of Health under award numbers R01AR053237 (AGR), R03AR057547 (DCG), and R01AR064255 (DCG). The content is solely the responsibility of the authors and does not necessarily represent the official views of the National Institutes of Health. This work was in part performed under the auspices of the U.S. Department of Energy by Lawrence Livermore National Laboratory under Contract DE-AC52-07NA27344. 


\section{REFERENCES}

[1] G.L. Semenza, Hydroxylation of HIF-1: oxygen sensing at the molecular level, Physiology (Bethesda). 19 (2004) 176-182. doi:10.1152/physiol.00001.2004.

[2] C. Wu, E.B. Rankin, L. Castellini, J.F. Alcudia, J. Fernandez-Alcudia, E.L. LaGory, et al., Oxygensensing PHDs regulate bone homeostasis through the modulation of osteoprotegerin, Genes Dev. 29 (2015) 817-831. doi:10.1101/gad.255000.114.

[3] Y. Wang, C. Wan, L. Deng, X. Liu, X. Cao, S.R. Gilbert, et al., The hypoxia-inducible factor alpha pathway couples angiogenesis to osteogenesis during skeletal development, J Clin Invest. 117 (2007) 1616-1626. doi:10.1172/JCI31581.

[4] M.B. Schaffler, O.D. Kennedy, Osteocyte signaling in bone, Current Osteoporosis Reports. 10 (2012) 118-125. doi:10.1007/s11914-012-0105-4.

[5] S.L. Dallas, M. Prideaux, L.F. Bonewald, The Osteocyte: An Endocrine Cell and More, Endocr Rev. (2013). doi:10.1210/er.2012-1026.

[6] J. Delgado-Calle, T. Bellido, Osteocytes and Skeletal Pathophysiology, Curr Mol Biol Rep. 1 (2015) 157-167. doi:10.1007/s40610-015-0026-y.

[7] T.S. Gross, K.A. King, N.A. Rabaia, P. Pathare, S. Srinivasan, Upregulation of osteopontin by osteocytes deprived of mechanical loading or oxygen, J Bone Miner Res. 20 (2005) 250-256. doi:10.1359/JBMR.041004.

[8] L.F. Raheja, L.F. Raheja, D.C. Genetos, C.E. Yellowley, Hypoxic osteocytes recruit human MSCs through an OPN/CD44-mediated pathway, 366 (2008) 1061-1066. doi:10.1016/j.bbrc.2007.12.076.

[9] E. Hinoi, H. Ochi, T. Takarada, E. Nakatani, T. lezaki, H. Nakajima, et al., Positive regulation of osteoclastic differentiation by growth differentiation factor 15 upregulated in osteocytic cells under hypoxia, J Bone Miner Res. 27 (2012) 938-949. doi:10.1002/jbmr.1538.

[10] A. Zahm, M. Bucaro, V. Srinivas, I. Shapiro, C. Adams, Oxygen tension regulates preosteocyte maturation and mineralization, Bone. 43 (2008) 25-31. doi:S8756-3282(08)00138-5 [pii]

10.1016/j.bone.2008.03.010.

[11] R.C. Riddle, J.M. Leslie, T.S. Gross, T.L. Clemens, Hypoxia-inducible factor-1a protein negatively regulates load-induced bone formation, J Biol Chem. 286 (2011) 44449-44456.

doi:10.1074/jbc.M111.276683.

[12] R.E. Tomlinson, M.J. Silva, HIF-1 $\alpha$ regulates bone formation after osteogenic mechanical loading, Bone. 73 (2015) 98-104. doi:10.1016/j.bone.2014.12.015.

[13] G.-L. Zuo, L.-F. Zhang, J. Qi, H. Kang, P. Jia, H. Chen, et al., Activation of HIFa Pathway in Mature Osteoblasts Disrupts the Integrity of the Osteocyte/Canalicular Network, PLoS ONE. 10 (2015) e0121266. doi:10.1371/journal.pone.0121266.

[14] Y. Lu, Y. Xie, S. Zhang, V. Dusevich, L.F. Bonewald, J.Q. Feng, DMP1-targeted Cre expression in odontoblasts and osteocytes, J Dent Res. 86 (2007) 320-325.

[15] V.H. Haase, J.N. Glickman, M. Socolovsky, R. Jaenisch, Vascular tumors in livers with targeted inactivation of the von Hippel-Lindau tumor suppressor, Proc Natl Acad Sci USA. 98 (2001) 15831588. doi:10.1073/pnas.98.4.1583.

[16] H.E. Ryan, M. Poloni, W. McNulty, D. Elson, M. Gassmann, J.M. Arbeit, et al., Hypoxia-inducible factor-1alpha is a positive factor in solid tumor growth, Cancer Res. 60 (2000) 4010-4015.

[17] S.L. Holmen, T.A. Giambernardi, C.R. Zylstra, B.D. Buckner-Berghuis, J.H. Resau, J.F. Hess, et al., Decreased BMD and limb deformities in mice carrying mutations in both Lrp5 and Lrp6, J Bone Miner Res. 19 (2004) 2033-2040. doi:10.1359/JBMR.040907.

[18] N.M. Collette, D.C. Genetos, A.N. Economides, L. Xie, M. Shahnazari, W. Yao, et al., Targeted deletion of Sost distal enhancer increases bone formation and bone mass, Proc Natl Acad Sci USA. 109 (2012) 14092-14097. doi:10.1073/pnas.1207188109.

[19] C.A. Toupadakis, A. Wong, D.C. Genetos, D.-J. Chung, D. Murugesh, M.J. Anderson, et al., Long-term administration of AMD3100, an antagonist of SDF-1/CXCR4 signaling, alters fracture repair, J Orthop Res. (2012). doi:10.1002/jor.22145.

[20] T.B. Bemenderfer, J.S. Harris, K.W. Condon, M.A. Kacena, Tips and Techniques for Processing and Sectioning Undecalcified Murine Bone Specimens, in: M.J. Hilton (Ed.), Skeletal Development and Repair, Humana Press, Totowa, NJ, 2014: pp. 123-147. doi:10.1007/978-1-62703-989-5_10.

[21] A.G. Robling, K.S. Kang, W.A. Bullock, W.H. Foster, D. Murugesh, G.G. Loots, et al., Sost, independent of the non-coding enhancer ECR5, is required for bone mechanoadaptation, Bone. 92 (2016) 180-188. doi:10.1016/j.bone.2016.09.001. 

adversely affects B-cell survival, J Bone Miner Res. 27 (2012) 1451-1461. doi:10.1002/jbmr.1608.

[23] V.J. Armstrong, M. Muzylak, A. Sunters, G. Zaman, L.K. Saxon, J.S. Price, et al., Wnt/beta-catenin signaling is a component of osteoblastic bone cell early responses to load-bearing and requires estrogen receptor alpha, J Biol Chem. 282 (2007) 20715-20727. doi:10.1074/jbc.M703224200.

[24] X. Tu, Y. Rhee, K. Condon, N. Bivi, M.R. Allen, D. Dwyer, et al., Sost downregulation and local Wnt signaling are required for the osteogenic response to mechanical loading, Bone. (2011). doi:10.1016/j.bone.2011.10.025.

[25] K. Sawakami, A.G. Robling, M. Ai, N.D. Pitner, D. Liu, S.J. Warden, et al., The Wnt co-receptor LRP5 is essential for skeletal mechanotransduction but not for the anabolic bone response to parathyroid hormone treatment, J Biol Chem. 281 (2006) 23698-23711. doi:10.1074/jbc.M601000200.

[26] B. Javaheri, A.R. Stern, N. Lara, M. Dallas, H. Zhao, Y. Liu, et al., Deletion of a single $\beta$-catenin allele in osteocytes abolishes the bone anabolic response to loading, J Bone Miner Res. 29 (2014) 705-715. doi:10.1002/jbmr.2064.

[27] X. Tu, J. Delgado-Calle, K.W. Condon, M. Maycas, H. Zhang, N. Carlesso, et al., Osteocytes mediate the anabolic actions of canonical Wnt/ $\beta$-catenin signaling in bone, Proc Natl Acad Sci USA. (2015) 201409857. doi:10.1073/pnas.1409857112.

[28] G.G. Loots, M. Kneissel, H. Keller, M. Baptist, J. Chang, N.M. Collette, et al., Genomic deletion of a long-range bone enhancer misregulates sclerostin in Van Buchem disease, Genome Research. 15 (2005) 928-935. doi:10.1101/gr.3437105.

[29] C.S. Yee, J.O. Manilay, J.C. Chang, N.R. Hum, D.K. Murugesh, J. Bajwa, et al., Conditional Deletion of Sost in MSC-Derived Lineages Identifies Specific Cell-Type Contributions to Bone Mass and B-Cell Development, J Bone Miner Res. 19 (2018) 179. doi:10.1002/jbmr.3467.

[30] N. Baumgarth, A Hard(y) Look at B-1 Cell Development and Function, J Immunol. 199 (2017) 33873394. doi:10.4049/jimmunol.1700943.

[31] C. Wan, S.R. Gilbert, Y. Wang, X. Cao, X. Shen, G. Ramaswamy, et al., Activation of the hypoxiainducible factor-1alpha pathway accelerates bone regeneration, Proc Natl Acad Sci USA. 105 (2008) 686-691. doi:10.1073/pnas.0708474105.

[32] A. Donneys, S.S. Deshpande, C.N. Tchanque-Fossuo, K.L. Johnson, J.T. Blough, J.E. Perosky, et al., Deferoxamine expedites consolidation during mandibular distraction osteogenesis, Bone. 55 (2013) 384-390. doi:10.1016/j.bone.2013.04.005.

[33] X. Shen, C. Wan, G. Ramaswamy, M. Mavalli, Y. Wang, C.L. Duvall, et al., Prolyl hydroxylase inhibitors increase neoangiogenesis and callus formation following femur fracture in mice, J Orthop Res. 27 (2009) 1298-1305. doi:10.1002/jor.20886.

[34] I. Kalajzic, B.G. Matthews, E. Torreggiani, M.A. Harris, P. Divieti Pajevic, S.E. Harris, In vitro and in vivo approaches to study osteocyte biology, Bone. 54 (2013) 296-306. doi:10.1016/j.bone.2012.09.040.

[35] J. Lim, J. Burclaff, G. He, J.C. Mills, F. Long, Unintended targeting of Dmp1-Cre reveals a critical role for Bmpr1a signaling in the gastrointestinal mesenchyme of adult mice, Bone Res. 5 (2017) 16049. doi:10.1038/boneres.2016.49.

[36] S.H. Shomento, C. Wan, X. Cao, M.-C. Faugere, M.L. Bouxsein, T.L. Clemens, et al., Hypoxiainducible factors 1alpha and 2alpha exert both distinct and overlapping functions in long bone development, J Cell Biochem. 109 (2010) 196-204. doi:10.1002/jcb.22396.

[37] H.C. St John, K.A. Bishop, M.B. Meyer, N.A. Benkusky, N. Leng, C. Kendziorski, et al., The Osteoblast to Osteocyte Transition: Epigenetic Changes and Response to the Vitamin D 3Hormone, Mol Endocrinol. (2014) me.2014-1091. doi:10.1210/me.2014-1091.

[38] D.C. Lee, H.A. Sohn, Z.-Y. Park, S. Oh, Y.K. Kang, K.-M. Lee, et al., A lactate-induced response to hypoxia, Cell. 161 (2015) 595-609. doi:10.1016/j.cell.2015.03.011.

[39] Y. Gong, R.B. Slee, N. Fukai, G. Rawadi, S. Roman-Roman, A.M. Reginato, et al., LDL receptorrelated protein 5 (LRP5) affects bone accrual and eye development, Cell. 107 (2001) 513-523.

[40] D.C. Genetos, C.A. Toupadakis, L.F. Raheja, A. Wong, S.E. Papanicolaou, D.P. Fyhrie, et al., Hypoxia decreases sclerostin expression and increases Wnt signaling in osteoblasts, J Cell Biochem. 110 (2010) 457-467. doi:10.1002/jcb.22559.

[41] D. Chen, Y. Li, Z. Zhou, C. Wu, Y. Xing, X. Zou, et al., HIF-1a Inhibits Wnt Signaling Pathway by Activating Sost Expression in Osteoblasts, PLoS ONE. 8 (2013) e65940-8. doi:10.1371/journal.pone.0065940. 
[42] T. Weng, Y. Xie, J. Huang, F. Luo, L. Yi, Q. He, et al., Inactivation of Vhl in osteochondral progenitor cells causes high bone mass phenotype and protects against age-related bone loss in adult mice, $\mathrm{J}$ Bone Miner Res. 29 (2014) 820-829. doi:10.1002/jbmr.2087.

[43] S. Movérare-Skrtic, P. Henning, X. Liu, K. Nagano, H. Saito, A.E. Börjesson, et al., Osteoblast-derived WNT16 represses osteoclastogenesis and prevents cortical bone fragility fractures, Nat Med. 20 (2014) 1279-1288. doi:10.1038/nm.3654.

[44] H.Y. Cho, H.J. Choi, H.J. Sun, J.-Y. Yang, J.H. An, S.W. Cho, et al., Transgenic mice overexpressing secreted frizzled-related proteins (sFRP)4 under the control of serum amyloid P promoter exhibit low bone mass but did not result in disturbed phosphate homeostasis, Bone. 47 (2010) 263-271. doi:10.1016/j.bone.2010.05.010.

[45] L.M. Calvi, G.B. Adams, K.W. Weibrecht, J.M. Weber, D.P. Olson, M.C. Knight, et al., Osteoblastic cells regulate the haematopoietic stem cell niche, Nature. 425 (2003) 841-846. doi:10.1038/nature02040.

[46] J. Zhu, R. Garrett, Y. Jung, Y. Zhang, N. Kim, J. Wang, et al., Osteoblasts support B-lymphocyte commitment and differentiation from hematopoietic stem cells, Blood. 109 (2007) 3706-3712. doi:10.1182/blood-2006-08-041384.

[47] J.Y. Wu, L.E. Purton, S.J. Rodda, M. Chen, L.S. Weinstein, A.P. McMahon, et al., Osteoblastic regulation of $B$ lymphopoiesis is mediated by $\mathrm{Gs}\{a$ alpha\}-dependent signaling pathways, Proc Natl Acad Sci USA. 105 (2008) 16976-16981. doi:10.1073/pnas.0802898105.

[48] K. Fulzele, D.S. Krause, C. Panaroni, V. Saini, K.J. Barry, X. Liu, et al., Myelopoiesis is regulated by osteocytes through Gsa-dependent signaling, Blood. 121 (2013) 930-939. doi:10.1182/blood-2012-06437160.

[49] L.M. Calvi, O. Bromberg, Y. Rhee, J.M. Weber, J.N.P. Smith, M.J. Basil, et al., Osteoblastic expansion induced by parathyroid hormone receptor signaling in murine osteocytes is not sufficient to increase hematopoietic stem cells, Blood. 119 (2012) 2489-2499. doi:10.1182/blood-2011-06-360933.

[50] Y. Fujiwara, M. Piemontese, Y. Liu, J.D. Thostenson, J. Xiong, C.A. O'Brien, RANKL (Receptor Activator of NFKB Ligand) Produced by Osteocytes Is Required for the Increase in B Cells and Bone Loss Caused by Estrogen Deficiency in Mice, J Biol Chem. 291 (2016) 24838-24850. doi:10.1074/jbc.M116.742452.

[51] M. Sato, N. Asada, Y. Kawano, K. Wakahashi, K. Minagawa, H. Kawano, et al., Osteocytes regulate primary lymphoid organs and fat metabolism, Cell Metab. 18 (2013) 749-758. doi:10.1016/j.cmet.2013.09.014.

[52] P. Divieti Pajevic, D.S. Krause, Osteocyte regulation of bone and blood, Bone. (2018). doi:10.1016/j.bone.2018.02.012. 


\section{FIGURE LEGENDS}

Figure 1. Inverse expression of HIF-1 $\alpha$ and HIF-2 $\alpha$ in osteocytic Vhl cKO mice. (A) Representative immunofluorescent staining for Vhl, HIF-1 $\alpha$, and HIF-2 $\alpha$ staining in femoral mid-diaphyseal cortical bone from cre-negative or osteocytic Vhl cKO mice. (B) Quantification of osteocytes staining positively for HIF-1 $\alpha$ and HIF-2 $\alpha$ in cre-negative or Vhl cKO femoral mid-diaphyseal cortical bone. $n=3-5$ mice per genotype; groups with different letters are statistically different from each other.

Figure 2. Systemic influence of osteocytic Vhl deletion. (A) Representative hematoxylin- and eosin-stained distal epiphysis from 16-week old male cre-negative and Vhl cKO mice. (B) Representative staining for the endothelial cell marker endomucin in the proximal metaphysis of cre-negative and $\mathrm{Vh} / \mathrm{cKO}$ mice. (C) microCT images illustrate the influence of osteocytic $V h /$ deletion on trabecular and cortical microarchitecture. Trabecular (D) bone volume fraction and (E) thickness of cre-negative and $\mathrm{Vhl}$ cKO mice. Quantification of femoral mid-diaphyseal cortical (F) tissue cross-sectional area, (G) cortical bone area, (H), cortical thickness, and (I) polar moment of inertia in cre-negative or Vh/ cKO mice. $n=7$ per genotype; groups with different letters are statistically different from each other.

Figure 3. Osteocytic Hif1a does not influence skeletal development. (A) microCT images of cortical and trabecular microarchitecture from cre-negative and osteocytic Hif1a cKO distal femora. (B) Distal metaphyseal trabecular bone volume fraction, (C) cortical area fraction, and (D) bone formation rate were each unaffected by deletion of osteocytic Hif1a. n=5-7 per genotype; groups with different letters are statistically different from each other.

Figure 4. Influence of $\mathrm{Vhl}$ on Wht signaling and requirement thereof for reversion of $\mathrm{Vhl} \mathbf{c K O} \mathrm{HBM}$ phenotype. Representative immunocytochemical staining for (A) sclerostin or activated $\beta$-catenin in femoral mid-diaphyseal cortical bone from cre-negative or $\mathrm{Vhl}$ cKO mice. (B) Representative microCT reconstruction of distal femora of cre-negative, $V h l \mathrm{cKO}, \mathrm{Vhl} \mathrm{cKO}$; $\mathrm{Lrp5} 5^{--}$or $\mathrm{Vhl} \mathrm{cKO}$; $S O S T^{T G}$ mice. Distal metaphyseal trabecular (C) bone volume fraction and (D) thickness or mid-diaphyseal cortical (E) thickness and (F) cortical area fraction of cre-negative, $\mathrm{Vhl} \mathrm{cKO}, \mathrm{Vhl} \mathrm{cKO}$; $\mathrm{Lrp5}^{--}$or $\mathrm{Vhl} \mathrm{cKO}$; $S O S T^{T G}$ mice. $\mathrm{n}=2-11$ mice per genotype; groups with different letters are statistically different from each other.

Figure 5. Influence of osteocytic $\mathrm{Vhl}$ deletion on B cell lymphopoiesis in bone marrow and spleen. (A) Femoral cortical marrow area as determined by $\mu \mathrm{CT}$. (B) Total numbers of bone marrow CD45+ hematopoietic cells. (C) Representative flow cytometry plots analyzing B cell developmental stages in control and Vhl cKO mice. (D) Bone marrow B cell precursor, immature, and mature B lymphocyte frequency in cre-negative and $\mathrm{Vhl}$ cKO mice. (E) Mean frequencies of LSK hematopoietic progenitors in the bone marrow and spleen of controls and Vhl cKO mice. (F) Gating strategy on live Cd11b-Gr1- cells for splenic B cell populations. (G) Mean frequencies of $\operatorname{lgM}^{-} \lg D^{-}$and $\operatorname{lgM}^{+} \lg D^{\text {high }}$ subsets within $C^{2} 19^{\text {int }} \mathrm{B}^{2} 20^{\text {int }}$ splenic B cells; (H) Mean frequencies of $\lg M^{-} \lg D^{-}$, $\operatorname{lgM}^{+} \lg D^{\text {low }}$, and $\operatorname{lgM}^{+/} \lg D^{\text {high }}$ subsets within $C D 19^{\text {int }} B 220^{\text {int }}$ splenic $B$ cells. $N=10$ mice per genotype; groups with different letters are statistically different from each other.

Supplemental Figure 1. (A) Representative negative control, without primary antibody, for immunocytochemistry. (B) L4 vertebrae from 16-week old male cre-negative or Vh/ cKO mice.

Supplemental Figure 2. Osteocytic Vhl cKO mice have improved skeletal microarchitecture. (A) Trabecular spacing, (B) trabecular number, and (C) cortical area fraction in 16-week old male cre-negative or $\mathrm{Vhl}$ cKO femora. (D) MacNeal's/Von Kossa staining of the undemineralized distal femur from cre-negative or $\mathrm{Vhl}$ cKO mice reveal abundant mineralized tissue (black) in mutant compared to control mice. (E) Toluidine blue staining of thin sections from the same groups panel $\mathbf{D}$ show that the dense mineralized tissue is bone and not calcified cartilage from the growth plate that failed to remodel. $n=7$ per genotype; groups with different letters are statistically different from each other.

Supplemental Figure 3. Absence of skeletal phenotype in osteocytic Hif1a cKO mice. Representative microCT images illustrate that deletion of osteocytic Hif1a has no influence on (A) trabecular number, (B) trabecular spacing, (C) cortical total area, (D) cortical bone area, (E) cortical thickness, (F) mineralizing surface per bone surface, $(\mathbf{G})$ endosteal and periosteal mineral apposition rate, or $(\mathbf{H})$ endosteal and periosteal bone 
formation rate. $\mathrm{n}=4-10$ animals per genotype; groups with different letters are statistically different from each other.

Supplemental Figure 4. Absence of apparent remodeling deficits in osteocytic Hif1a cKO mice. (A) Tissue mineralization in cre-negative or Hif1a cKO mice, visualized by MacNeal's/Von Kossa; (B) TRAPstained osteoclasts were found in both cre-negative and Hif1a cKO sections.

Supplemental Figure 5. Trabecular and cortical microarchitecture in compound VhIWnt signaling mutant mice. (A) Quantification of osteocytes staining positively for sclerostin or activated $\beta$-catenin in crenegative or $\mathrm{Vhl}$ cKO femoral mid-diaphyseal cortical bone.Quantification of (B) trabecular number and (C) trabecular spacing in femoral distal metaphysis of mice with indicated genotypes. (D) cortical area fraction and (E) polar moment of inertia in mid-diaphyseal femoral cortical bone of mice with indicated genotypes. $n=2-6$ animals per genotype; groups with different letters are statistically different from each other.

Supplemental Figure 6. B cell development in bone marrow and spleen of cre-negative and osteocytic Vhl cKO mice. (A) Absolute numbers of B cell precursors, immature and mature B lymphocytes in the bone marrow of control and $\mathrm{Vhl}$ cKO mice. Total number of LSK cells in (B) bone marrow and (C) spleens of crenegative and $\mathrm{Vh} / \mathrm{cKO}$ mice. Groups with different letters are statistically different from each other 


\begin{tabular}{|c|c|c|}
\hline $\begin{array}{c}\text { TABLE } 1 . \\
\text { CD45 } \\
\text { (mean } \% \pm \text { SD) }\end{array}$ & $\begin{array}{l}\text { Control } \\
(n=10)\end{array}$ & $\begin{array}{c}\text { Vhl cKO } \\
(n=10)\end{array}$ \\
\hline \multicolumn{3}{|l|}{ Bone marrow } \\
\hline $\begin{array}{l}\text { B lymphocytes } \\
\text { (total CD19+) }\end{array}$ & $26.76 \pm 8.38$ & $12.85 \pm 3.25$ *** \\
\hline $\begin{array}{l}\text { T lymphocytes } \\
\text { (total CD3+) }\end{array}$ & $1.54 \pm .0 .85$ & $1.67 \pm .1 .98$ \\
\hline $\begin{array}{l}\text { Monocytes } \\
\left(\mathrm{CD} 11 \mathrm{~b}+\mathrm{Gr} 1^{\text {low }}\right)\end{array}$ & $5.12 \pm .1 .75$ & $6.70 \pm .1 .38^{\star}$ \\
\hline $\begin{array}{l}\text { Granulocytes } \\
\left(C D 11 b+G r 1^{\text {high }}\right)\end{array}$ & $55.77 \pm .7 .80$ & $66.46 \pm .5 .34^{\star \star}$ \\
\hline \multicolumn{3}{|l|}{ Spleen } \\
\hline $\begin{array}{l}\text { B lymphocytes } \\
\text { (total CD19+) }\end{array}$ & $59.51 \pm .7 .62$ & $37.24 \pm .13 .28^{\star \star \star}$ \\
\hline $\begin{array}{l}\text { T lymphocytes } \\
\text { (total CD3+) }\end{array}$ & $23.84 \pm .2 .80$ & $20.04 \pm .8 .30$ \\
\hline $\begin{array}{l}\text { Monocytes } \\
\left(\mathrm{CD} 11 \mathrm{~b}+\mathrm{Gr} 1^{\text {low }}\right)\end{array}$ & $2.93 \pm .0 .42$ & $4.47 \pm .1 .06^{\star \star \star}$ \\
\hline $\begin{array}{l}\text { Granulocytes } \\
\left(C D 11 b+G r 1^{\text {high }}\right)\end{array}$ & $2.09 \pm .0 .59$ & $12.34 \pm .8 .51^{\star \star}$ \\
\hline
\end{tabular}

${ }^{*} p<0.05,{ }^{* *} p<0.01,{ }^{\ddagger} p=0.06$ 


\section{ACCEPTED MANUSCRIPT}

\section{HIGHLIGHTS}

- Osteocytes - the terminally differentiated cells in bone-exert a robust influence on bone mass through their capacity to perceive pericellular oxygen.

- In contrast to reports using osteoprogenitors, osteocytes do not require Hif1a, suggesting a differentiationdependent need for and use of HIF-alpha subunits.

- Influencing oxygen availability drives canonical Wnt signaling in bone, a current target of pharmacologic intervention to improve bone mass and prevent age-related osteoporosis.

- Absence of osteocytic Vhl reduces B cell maturation in bone marrow. 
A cre-negative

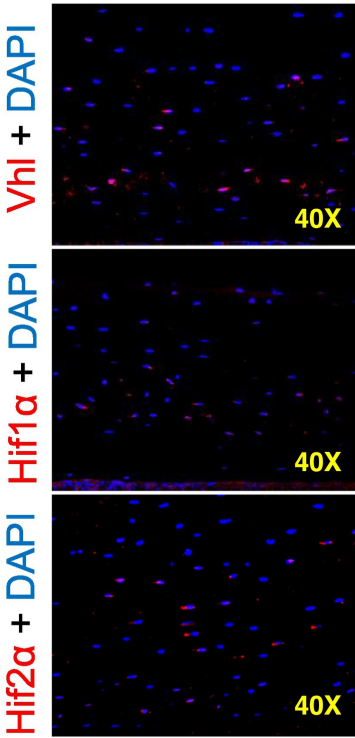

Vhl cKO
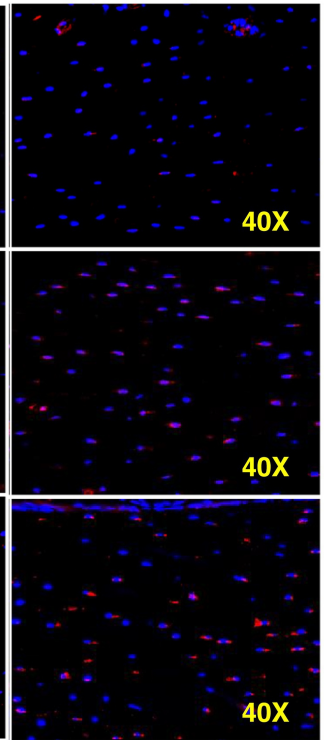

B

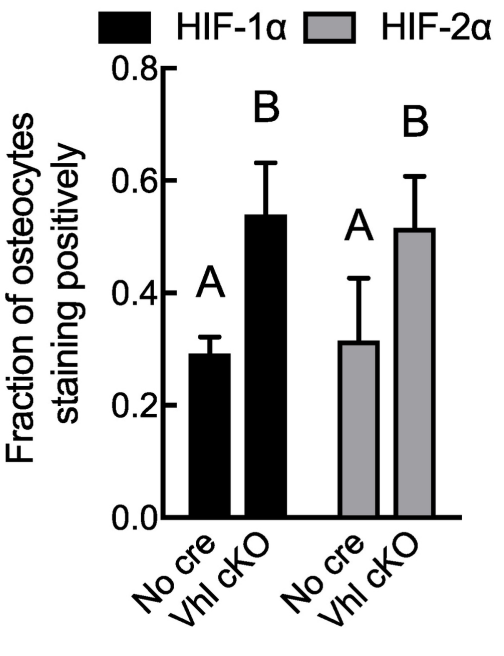

Figure 1 
A cre-negative

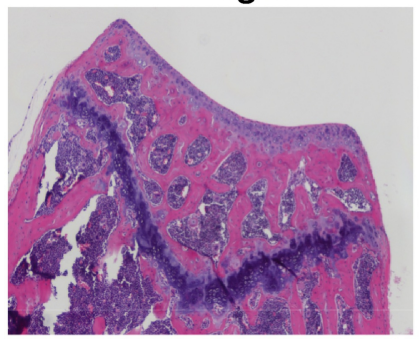

C

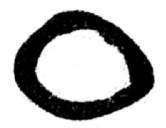

cre-negative

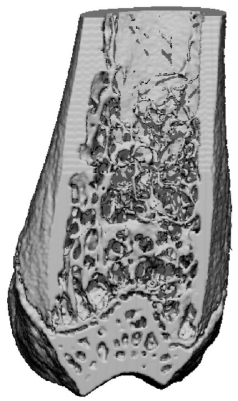

Vhl cKO
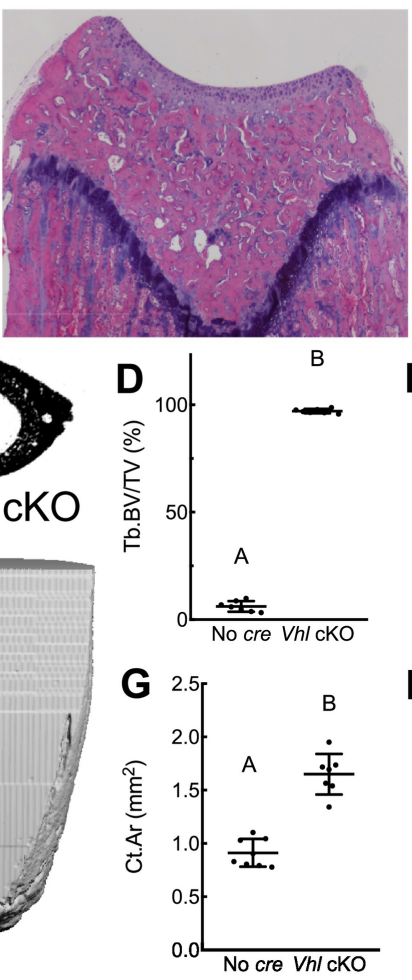

B cre-negative
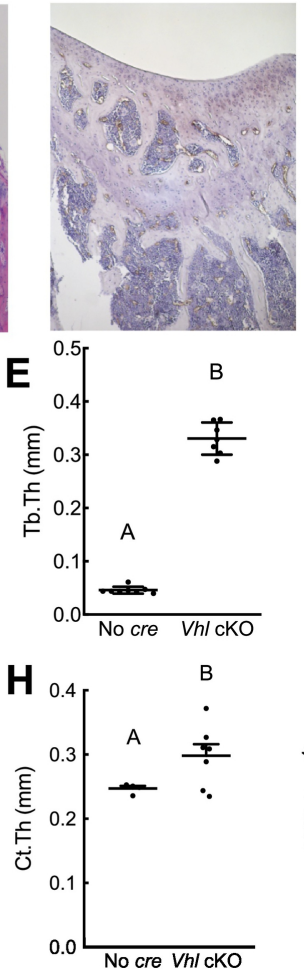

Vhl cKO
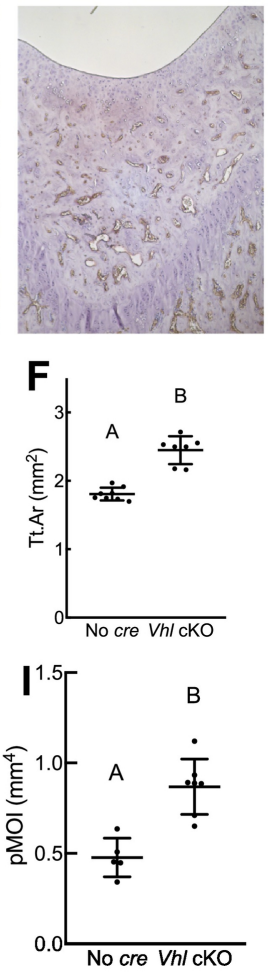

Figure 2 


\section{A cre-negative Hif1a cKO $\mathbf{B}$}
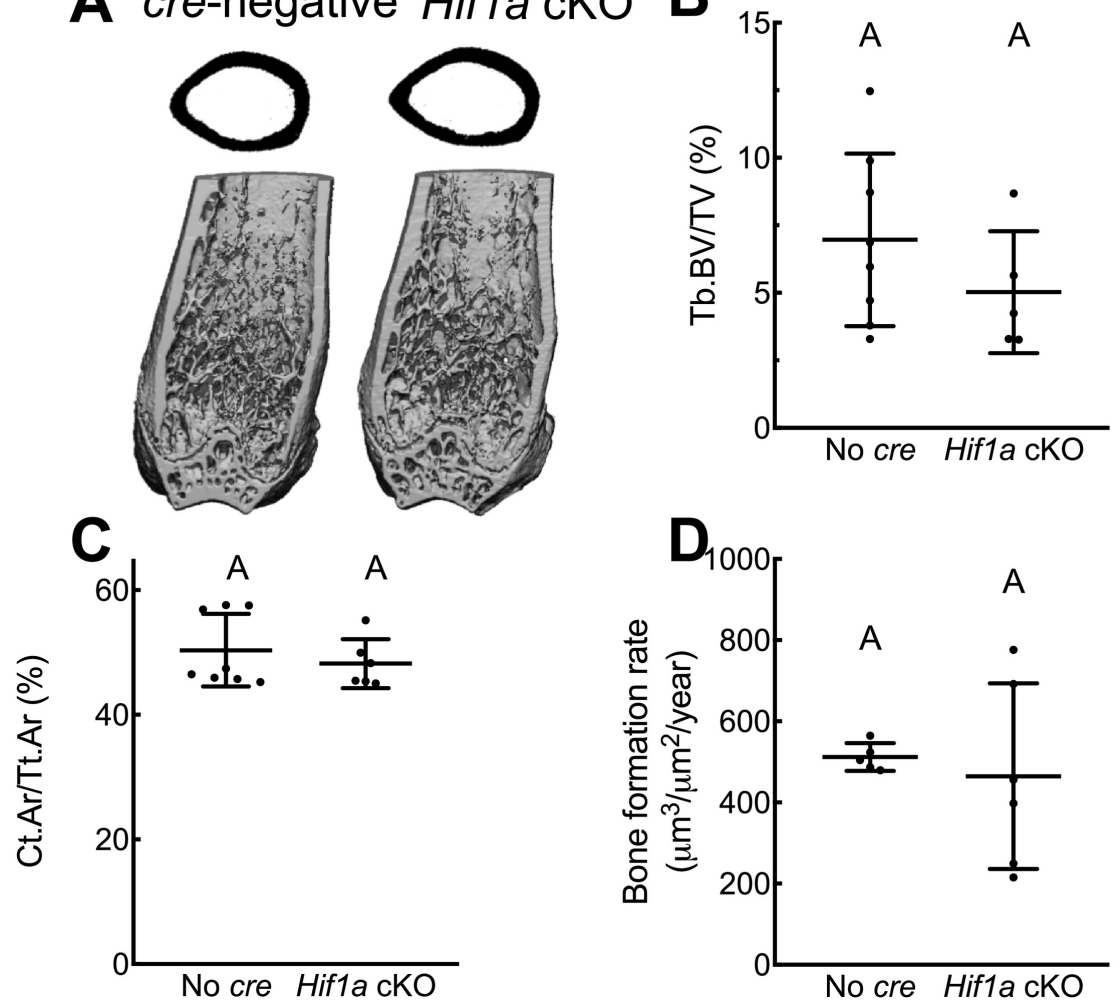

Figure 3 

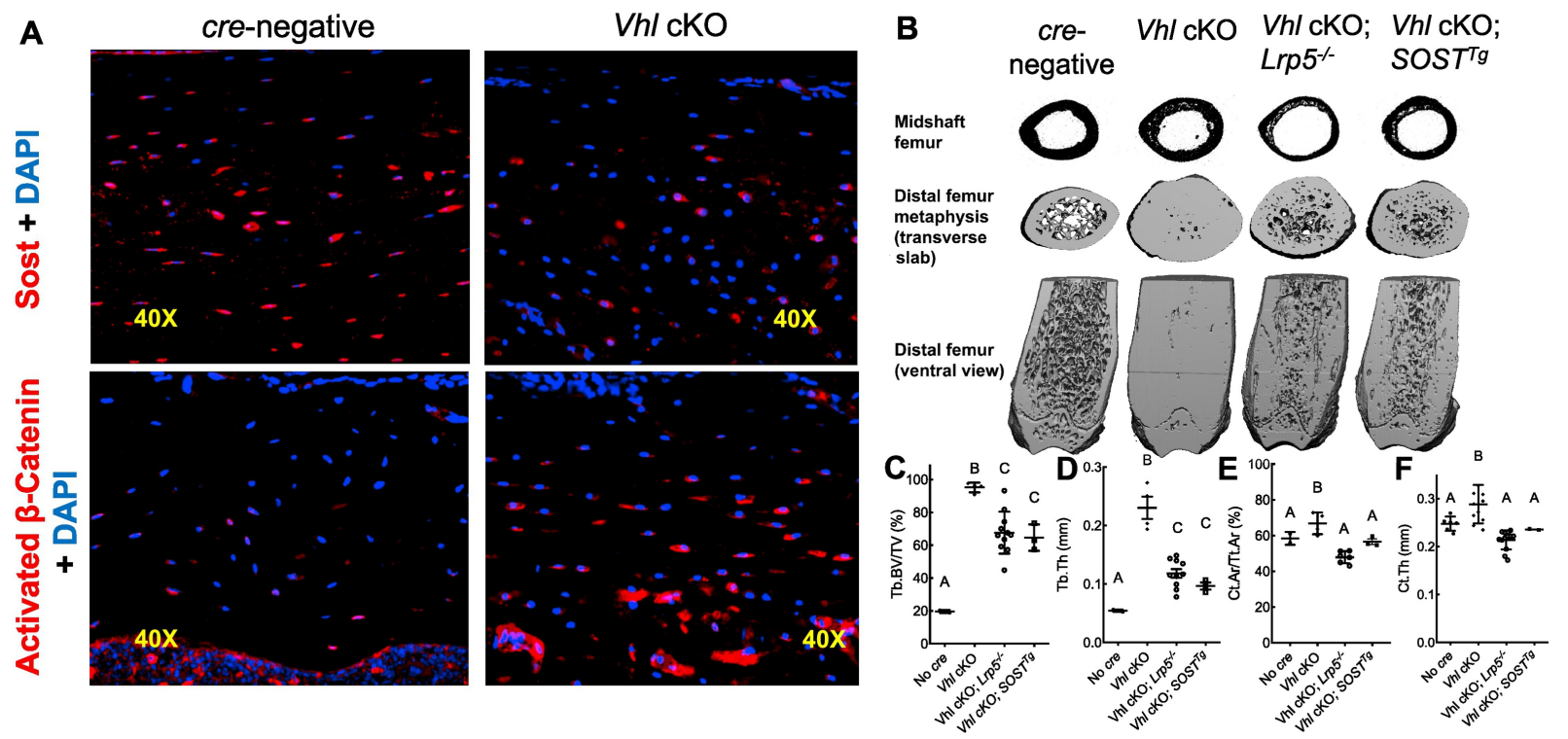

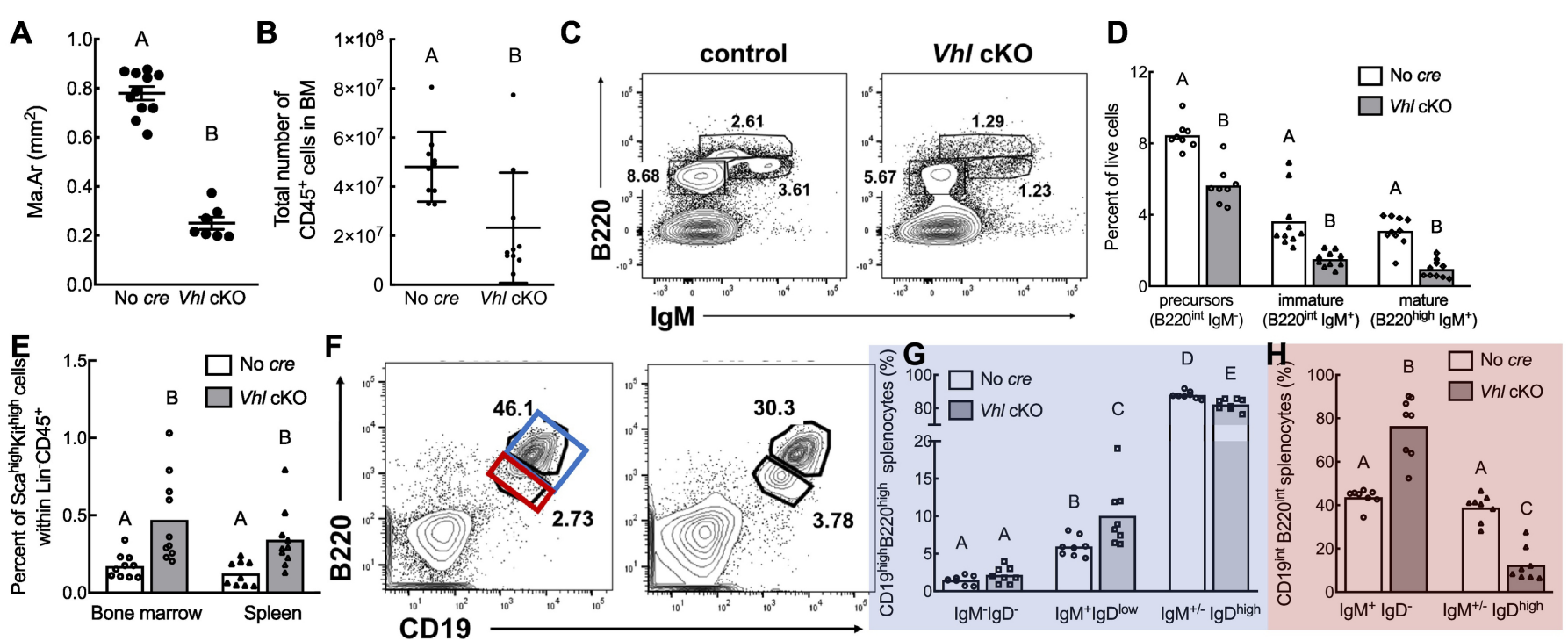

Figure 5 